\title{
Monitoring of Oocyte/Embryo Respiration Using Electrochemical-Based Oxygen Sensors
}

\author{
Yusra Obeidat ${ }^{1}$, Amanda Evans ${ }^{2}$, William Tedjo ${ }^{1,3}$, Adam Chicco ${ }^{2,3}$, Elaine Carnevale ${ }^{2}$ \\ and Tom Chen $1,3, *$ \\ 1 Department of Electrical and Computer Engineering, Colorado State University, Fort Collins, CO 80523, \\ USA. \\ 2 Department of Biomedical Sciences, Colorado State University, Fort Collins, CO 80523, USA. \\ 3 School of Biomedical Engineering, Colorado State University, Fort Collins, CO 80523, USA. \\ * Correspondence: Thomas.chen@colostate.edu; Tel: 970-491-6574
}

\begin{abstract}
Current commercially available instruments for monitoring mitochondrial respiration are incapable of single cell measurements. Therefore, we developed a three-electrode, Clark-type biosensor suitable for mitochondrial respirometry in single oocytes and embryos. The biosensor was embedded in a PMMA (polymethyl methacrylate) micro-chamber to allow investigation of single oocytes/embryos immersed in up to $100 \mu \mathrm{L}$ of respiration buffer. The micro-chamber was completely sealed to avoid oxygen exchange between the inside of the chamber and the atmosphere, while being maintained at a temperature of $38.5^{\circ} \mathrm{C}$ to preserve cell viability. Using amperometry, the oxygen consumption of cells inside the micro-chamber was measured as a change in output current and converted to femto-mol (fmol) oxygen consumed per second based on calibrations with known buffer oxygen concentrations. The sensor measured basal cell respiration supported by endogenous substrates, respiration associated with proton leak induced by inhibition of the adenosine triphosphate (ATP) synthase (complex V) with oligomycin, and the maximal noncoupled respiratory capacity revealed by Carbonyl cyanide-4-(trifluoromethoxy)phenylhydrazone (FCCP) titration. Some potential applications of this oxygen sensor system include evaluating effects of metabolic therapies on oocyte bioenergetics, and monitoring mitochondrial function throughout oocyte maturation and blastocyst development to predict embryo viability to compliment assisted reproductive technologies.
\end{abstract}

Keywords: Clark-type oxygen sensor; mitochondrial respiration; amperometry; cyclic voltammetry; oocyte; embryo

\section{Introduction}

Mitochondrial respiration is an important indicator of cell activity and viability that is closely associated with organismal metabolic health and disease $[1,2]$. Cellular oxygen consumption is linked to energy metabolism by oxidative phosphorylation, the coupling of metabolic substrate oxidation to the production of adenosine triphosphate (ATP) in mitochondria. Accordingly, the rate of basal cell respiration is influenced by cellular energy demand and is typically below mitochondrial respiratory capacity. Respiration is not perfectly coupled to ATP synthesis, as it can result in part from proton "leak" across the inner mitochondrial membrane [3]. Therefore, it is important to distinguish basal respiration from "leak" and maximal respiratory capacities when evaluating cellular respiration, which also provides indices of oxidative phosphorylation efficiency and respiratory reserve capacity relevant to bioenergetic function [4]. Leak respiration is routinely induced by inhibiting ATP synthase with oligomycin, while the maximal respiratory capacity can be revealed by titration of an uncoupler such as carbonyl cyanide-4-(trifluoromethoxy) phenylhydrazone (FCCP) [5]. 
The oocyte is a unique cell that is challenging to study due to its size, structure, associated cells and paucity especially in some species as horses and humans where it is much more difficult to get oocytes from them than mice and cattle [6,7]. Energy production by oocyte mitochondria is critical for oocyte maturation, spindle alignment, fertilization and early embryo development $[3,8]$. Research by our group and others has been performed to better understand mitochondrial activities of oocytes during maturation and in response to different stimuli that affect oocyte viability [8-10]. Comparing to the existing method of using morphological assessment for oocyte/embryo quality [11], the ability to measure mitochondrial respiration of single oocyte under different stimuli will enable better understanding of oocyte and embryo physiology and provide metrics for better prediction of oocyte/embryo quality for applications, such as assisted reproduction technologies [9].

The use of oxygen sensors for in-vitro monitoring of cell activities and for determining the effect of drugs on cell metabolism has been demonstrated using optical techniques such as florescence quenching by measuring the change in oxygen concentration near the cell [12, 13]. This method has drawbacks such as temperature sensitivity, and it requires a long time to reach stability [13, 14]. Scanning electrochemical microscopy (SECM) has been used to measure oxygen concentration of a given sample. Edwards et al. investigated the biochemical applications of SECM and its required instrumentation and theoretical modelling to conclude that SECM-based methods have wide applications in biological systems, providing improved understanding of many biological phenomena, with an emphasis on living systems [15]. These methods can be developed to examine cellular processes at a single-cell level. Shiku et al. used SECM for measuring the oxygen consumption of single bovine embryos to compare respiration rates at various stages of development including the morula and blastocyst [11]. More recently, Sugimura et al. used SECM to measure the respiration rate in individual bovine oocytes by scanning a micro-disc Pt electrode back and forth between the bulk solution and sample surface [16]. The respiration rate was calculated by finding the oxygen concentration difference between the two sites and estimated using the spherical diffusion theory [17]. SECM was also used with a time-lapse imaging system in EmbryoScope [18]. Tejera et al. used EmbryoScope to study the effect of different types of stimulation on human oocyte respiration and to understand the relationship between the respiration activity of the oocytes and their reproductive outcome [9]. More recently they used the same system to find the correlation between oxygen consumption (OC) measurements at different stages of embryo development, measuring OC during embryo cleavages and combining that information with morphokinetics to assess implantation potential [10]. The SECM technology provides a relatively simple electrochemical control and measurement, in addition to the feasible integration with other techniques such as atomic force microscopy (AFM) and optical microscopy to obtain both electrochemical and topographical information [19]. However, SECM is bulky and requires expensive instruments for high resolution, such as piezoelectric positioners [20], stepper motors to control the position of the tip in the $x-y$ plane [21] parallel to the interface of interest, and a large volume of sample medium in the $\mathrm{mL}$ range [15].

The Clark-type oxygen sensors [22] use the principle of electrochemistry [23] for oxygen measurement. Dissolved oxygen sensors operate by electrochemical reduction of the oxygen particles dissolved in the sample solution and measurement of the reduction current proportional to the oxygen concentration [24]. Clark sensors typically consist of three electrodes: working (WE), reference (RE) and counter (CE). Internal electrolyte within the electrochemical cell provides a closed electrical loop for the WE and CE to allow potential control within the electrochemical cell. To improve the selectivity and avoid contamination of electrodes by the samples, an oxygen permeable membrane is overlaid on the electrodes, and usually a reservoir of sample solution is located on top of the oxygen permeable membrane to contain the sample solution under test. The mode of operation of the Clark sensor is amperometric $[25,26]$. The dissolved oxygen is brought to the surface of WE and a negative voltage is applied between the WE and CE. Electrochemical reduction happens at the $\mathrm{WE}$, and the reduction current of oxygen is proportional to the dissolved oxygen of the sample solution as shown in Figure 1. 


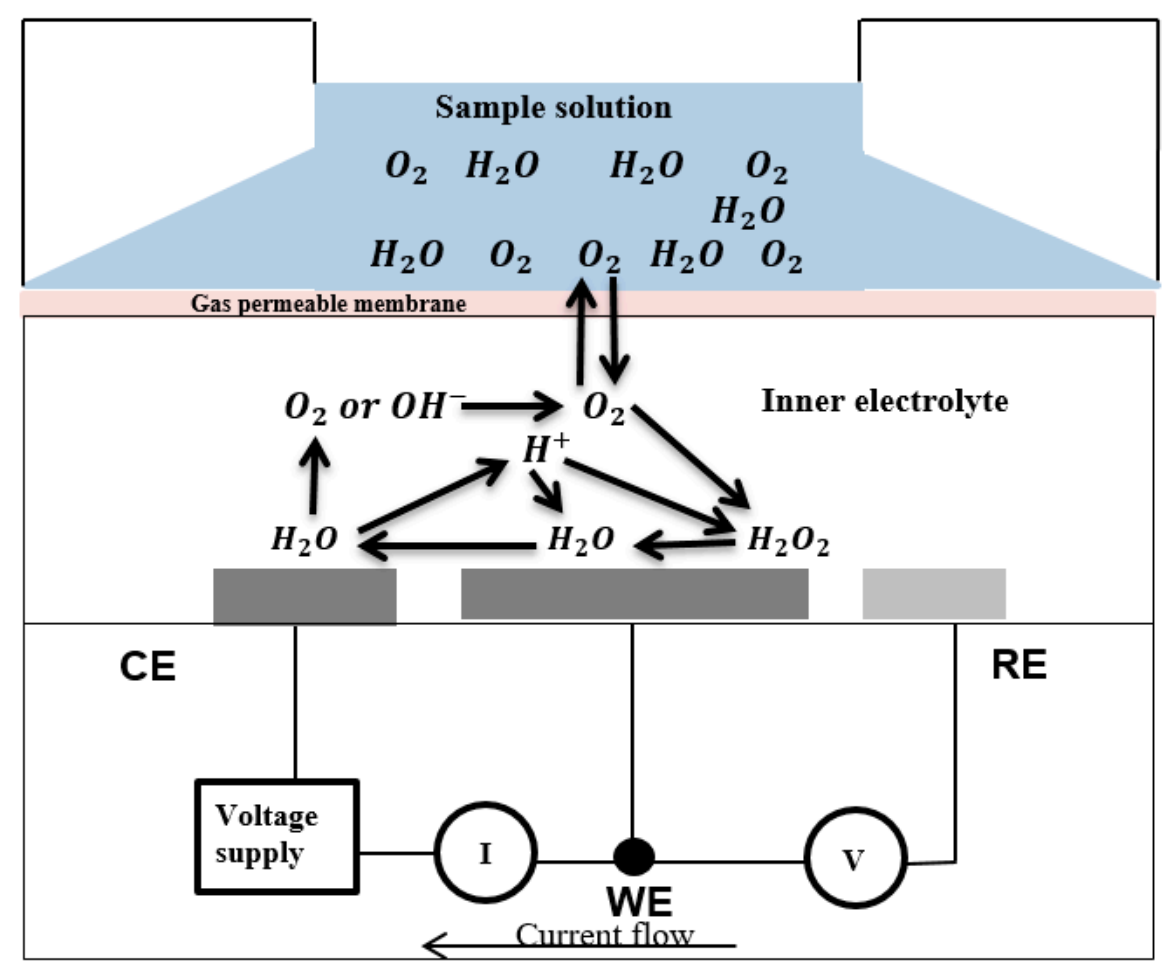

Figure 1. Principle of Clark sensor operation [2].

The reduction at the WE is represented by the following equation $[25,27]$ :

$$
\begin{gathered}
\mathrm{O}_{2}+2 \mathrm{H}^{+}+2 e^{-} \rightarrow \mathrm{H}_{2} \mathrm{O}_{2} \\
\mathrm{H}_{2} \mathrm{O}_{2}+2 \mathrm{H}^{+}+2 e^{-} \rightarrow 2 \mathrm{H}_{2} \mathrm{O}
\end{gathered}
$$

The resultant passes through the electrolyte to the CE and oxidizes as following [28]:

$2 \mathrm{H}_{2} \mathrm{O} \rightarrow \mathrm{O}_{2}+4 \mathrm{H}^{+}+4 e^{-}$

Also, the oxygen reduction current is related to the oxygen partial concentration is related by the following equation [26,29]:

$I(t)=n F A P_{m}\left(\frac{\partial C}{\partial x}\right), @ x=0$

Where $\mathrm{n}$ is the number of electrons, $\mathrm{F}$ is Faraday's constant, A is surface area of the working electrode, and $\mathrm{P}_{\mathrm{m}}$ is permeability of the membrane.

One of the distinctive feature of Clark sensors is that they can be very small in size, making them more suitable for measuring single cell respiration. Clark sensor has many applications in biosensor development, clinical analysis and fermentation monitoring [2, 26]. Miyahara et al. characterized size, linearity, selectivity, reproducibility, and response time as some of the main properties to determine the ability of the Clark sensor to measure dissolve oxygen [29]. Various types of Clark-type oxygen sensors with three or two electrodes have been developed in the last few years. Suzuki et al. used a micromachining technique to fabricate a miniature Clark oxygen electrode on a glass substrate with a silver working electrode, gold counter electrode and $\mathrm{Ag} / \mathrm{AgCl}$ reference electrode to demonstrate the properties of three electrodes system in measuring dissolved oxygen. The electrode shows a good linearity and fast response time [30]. Jobset et al. demonstrated the use of the thin-film technology to produce a planar miniaturized Clark-type oxygen sensor. The sensor 
consists of platinum working and counter electrodes and $\mathrm{Ag} / \mathrm{AgCl}$ reference electrode covered with a photo-structured hydrogel layer to form electrolyte compartment, and a photo-structured hydrophobic gas-permeable membrane to minimize the effects of fouling on sensor response. The sensor was shown to have excellent performance and no effects of fouling on sensor response when it was tested in buffer solutions and bovine serum [25]. Miyahara et al. demonstrated the use of a printing method to design planar amperometric oxygen sensor with a stable response and good linearity. The sensor consists of four glass substrates stuck together by epoxy resin. A Pt cathode and an Ag anode are deposited on one of these glass substrates by RF sputtering and by printing, respectively. The structure has an internal electrolyte solution and is sealed off by an oxygenpermeable membrane. The sensor was shown to have good linearity and stable response for more than 2000 hours of operation [29]. McLaughlin et al. investigated the design of a microfabricated oxygen sensor with a microfabricated thin-film electrode matrix overlaid with a solid-state proton conductive matrix (PCM) and encapsulated in a bio-inert polytetrafluoroethylene (PTFE) film to isolate the electrodes from media and to prevent interference signals from other electroactive species. The sensor was shown to have a good linearity and fast response time [31]. Wu et al. used a novel fabrication technique to make miniature Clark-type oxygen sensor integrated with a microstructure to measure the oxygen consumption of different concentrations of E. coli bacteria. The oxygen chip consists of a glass substrate with a three-electrode configuration and a (polydimethylsiloxane) (PDMS) container with an immobilized PDMS oxygen-permeable membrane. The miniature sensor has shown to have a fast response time of 6.8 seconds, good linearity, and a long lifetime with the ability to be integrated with a microfluidic system [26]. More recently, Obeidat and Chen investigated the response characteristics of a three-electrode, Clark-type, oxygen sensor using microelectrodes under a variety of operating conditions. The silicon based microelectrodes were fabricated using a complementary metal oxide semiconductor (CMOS) process with Pt surface using a lift-off process. The device was shown to have a good linearity and fast response time of 2-4 seconds [32].

The main aspects that need to be taken into consideration in designing a reliable sensor include the fabrication of the electrodes in such a way that reduces the electrochemical crosstalk between electrodes, a stable reference electrode and long life time, good linearity, good specificity and reproducibility, durability and bonding properties of the oxygen permeable membrane, and fixing the characteristics of the internal electrolyte to easily integrate with other sensor for multiplexed applications [26, 27]. One of the main difficulties in integrating the Clark sensor with other sensors is the internal electrolyte solution which is incompatible with the fabrication processes [31,33]. To improve electrical conductivity and eliminate the need for rehydration, Niazi et al. designed a microelectrode with solid electrolyte membrane [33]. Jobst et al. used a solid-state proton conductive matrix PCM to increase a sensor's lifetime and to eliminate the need for rehydration [25]. In our previous work [32], four conditions for measuring the characteristics of an oxygen sensor using three Pt microelectrodes were characterized. The best linearity and sensitivity was achieved when Nafion was used as solid-state electrolyte and oxygen-permeable membrane. Our results also verified that comparable linearity and sensitivity can be achieved using Nafion as solid-state electrolyte as well as membrane.

Miniaturization of Clark sensors has opened the door for precise evaluation of cellular oxygen consumption integrated within a cell culture platform. This facilitates screening of potential therapeutic compounds that target metabolism on single cells, significantly reducing the material and financial costs associated with other approaches [34]. In this paper, a Clark-type oxygen sensor and a micro-environment were developed to assess mitochondrial respiratory function in single equine or bovine oocytes and bovine embryos. Validity, reproducibility and stability of the signal were demonstrated by repeating sample titrations and generating data over continuous periods up to 120 minutes, with expected reductions in basal respiration in the presence of ATP synthase inhibition. Furthermore, we demonstrated for the first time in single cell measurements that careful titration of an uncoupler is necessary to reveal the maximal respiratory capacity of oocytes/embryos and avoid erroneous respiratory inhibition. Potential future applications of this sensor system include the 
evaluation of metabolic therapies on oocyte bioenergetics and the monitoring of mitochondrial function throughout oocyte maturation and blastocyst development to predict embryo viability in the context of assisted reproductive technologies.

\section{Materials and Methods}

\subsection{Sensor Electrodes}

All experiments used the screen-printed electrodes DS550 (Dropsens, Llanera, Spain). The electrodes were printed on ceramic substrate of $33 \mathrm{~mm}$ length, $10 \mathrm{~mm}$ width, and $0.5 \mathrm{~mm}$ height. The electrochemical cell consists of: circular Pt WE of $4 \mathrm{~mm}$ diameter, curved $\mathrm{Pt} \mathrm{CE}$, and small curved silver RE, and silver electric contact as shown in Figure 2.

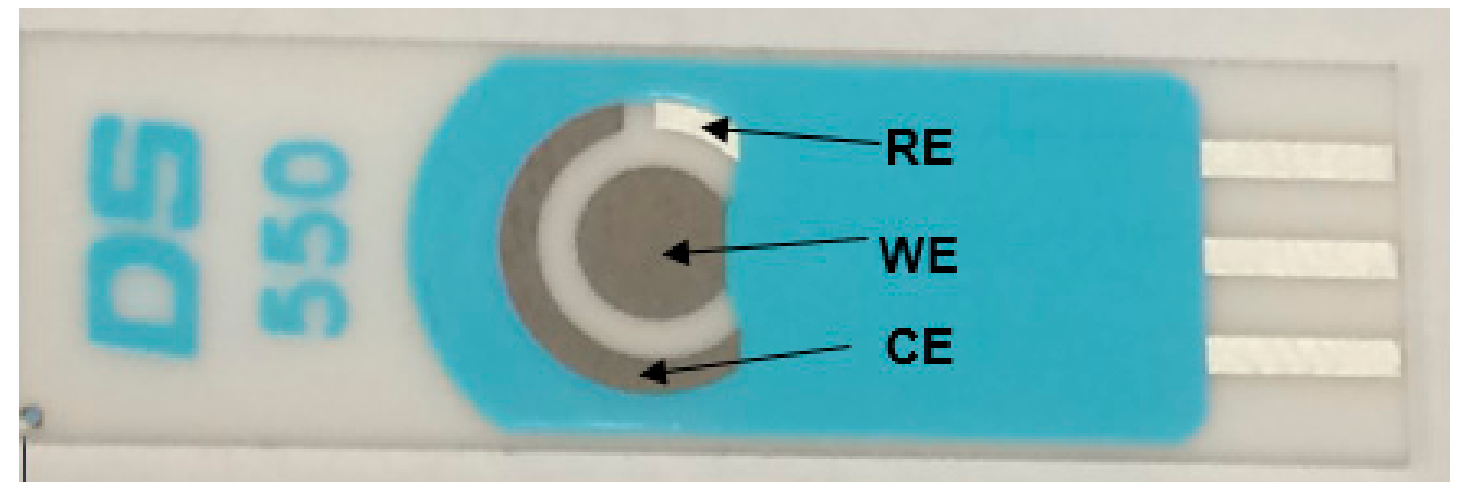

Figure 2. DS550 screen printed electrodes.

\subsection{Sensor Surface Modification}

Among different surface preparation methods, Nafion was chosen as the solid-state electrolyte as well as the membrane. Using Nafion only is more compatible with integration and multiplexed sensing applications than using other electrolytes and membranes [32]. Nafion perfluorinated resin was purchased from Sigma Aldrich (St. Louis, Missouri), and the WE of the chip was covered by a thin layer of Nafion before use.

\subsection{Oocytes and Embryos}

Equine and bovine oocytes were collected from live mares or bovine ovaries from a slaughterhouse. Equine oocytes were matured in Tissue Culture Medium 199 (Gibco ${ }^{\mathrm{TM}}$, Grand Island, NY) with additions of $10 \%$ fetal calf serum, $0.2 \mathrm{mM}$ pyruvate and $25 \mu \mathrm{g} / \mathrm{ml}$ gentamicin, with or without the addition of hormones and growth factors $(15 \mathrm{ng} / \mathrm{ml} \mathrm{FSH}, 1 \mu \mathrm{g} / \mathrm{ml} \mathrm{LH}, 1 \mu \mathrm{g} / \mathrm{ml} \mathrm{E}$, 200 $\mu \mathrm{g} / \mathrm{ml} \mathrm{P} 4,10 \mathrm{ng} / \mathrm{ml}$ IGF and $100 \mathrm{ng} / \mathrm{ml}$ EGF) depending on the degree of oocyte maturation. Equine oocyte maturation was performed in an atmosphere of $6 \% \mathrm{CO}_{2}$ at $38.2{ }^{\circ} \mathrm{C}$. Bovine oocyte maturation, fertilization and embryo culture were performed as previously described and using CDM1/CD/M2 sequential embryo culture media [35]. Prior to analyses, oocytes or embryos were transported $<20$ min to another laboratory in medium TCM 199 with Hanks' salts, $10 \%$ newborn calf serum, $0.2 \mathrm{mM}$ pyruvate and $25 \mu \mathrm{g} / \mathrm{ml}$ gentamicin) while housed within an insulated container.

\subsection{Respiration Buffers}

Mitochondrial respiration medium Mir05 (respiration buffer) was prepared at Colorado State University as previously described [4], with contents listed in Table 1. 
Table 1. Respiration Buffer Contents

\begin{tabular}{ll}
\hline Content & Company \\
\hline $0.5 \mathrm{mM} \mathrm{EGTA}$ & Sigma E 4378 \\
$3 \mathrm{mM} \mathrm{MgCl}_{2} .6 \mathrm{H}_{2} \mathrm{O}$ & Scharlau MA 0036
\end{tabular}

60mM Lactobionic acid

20mM Taurine

$\mathrm{KH}_{2} \mathrm{PO}_{4}$

20mM HEPES

110mM D-Sucrose

1g/l Bovine Serum Albumin, essentially

fatty acid free, Fraction $\mathrm{V}$
Adrich 153516

Sigma T 0625

Merck 104873

Sigma H7523

Roth 4621.1 or Sigma 84097

Sigma A 6003

\subsection{Stimuli for Oocytes and Embryos}

Carbonyl cyanide-4-(trifluoromethoxy) phenylhydrazone (FCCP; 1-4 $\mu \mathrm{M}$ ) was purchased from Sigma Aldrich (St. Louis, Missouri) and was used as a potent protonophore uncoupler of mitochondrial oxidative phosphorylation. Oligomycin $(1 \mu \mathrm{M})$ was purchased from Sigma Aldrich (St. Louis, Missouri) and was used as ATP synthase inhibitor. Digitonin was brought from Sigma Aldrich (St. Louis, Missouri) and was used to permeabilize the cell membrane so fuels can get into the cell.

\subsection{Micro-Chamber}

A micro-chamber for housing single oocyte and embryo cells was constructed using PMMA (polymethyl methacrylate) with a slit to allow the electrode chip to be mounted as shown in Figure 3. The screen-printed sensor chip was placed on a flat PMMA base, overlaid on top by the coneshaped micro-chamber. The assembly between the base and the cone-shaped micro-chamber is sealed by a rubber ring. The cone shaped micro-chamber provides a volume for up to $100 \mu \mathrm{L}$ of buffer and has a $2 \mathrm{~mm}$ diameter top opening to allow loading of buffer and oocyte/embryo cells into the chamber. Paraffin oil (Sigma Aldrich,St. Louis, Missouri) was used to seal the micro-chamber to prevent atmospheric oxygen from diffusing into the micro-chamber during the experiments. 


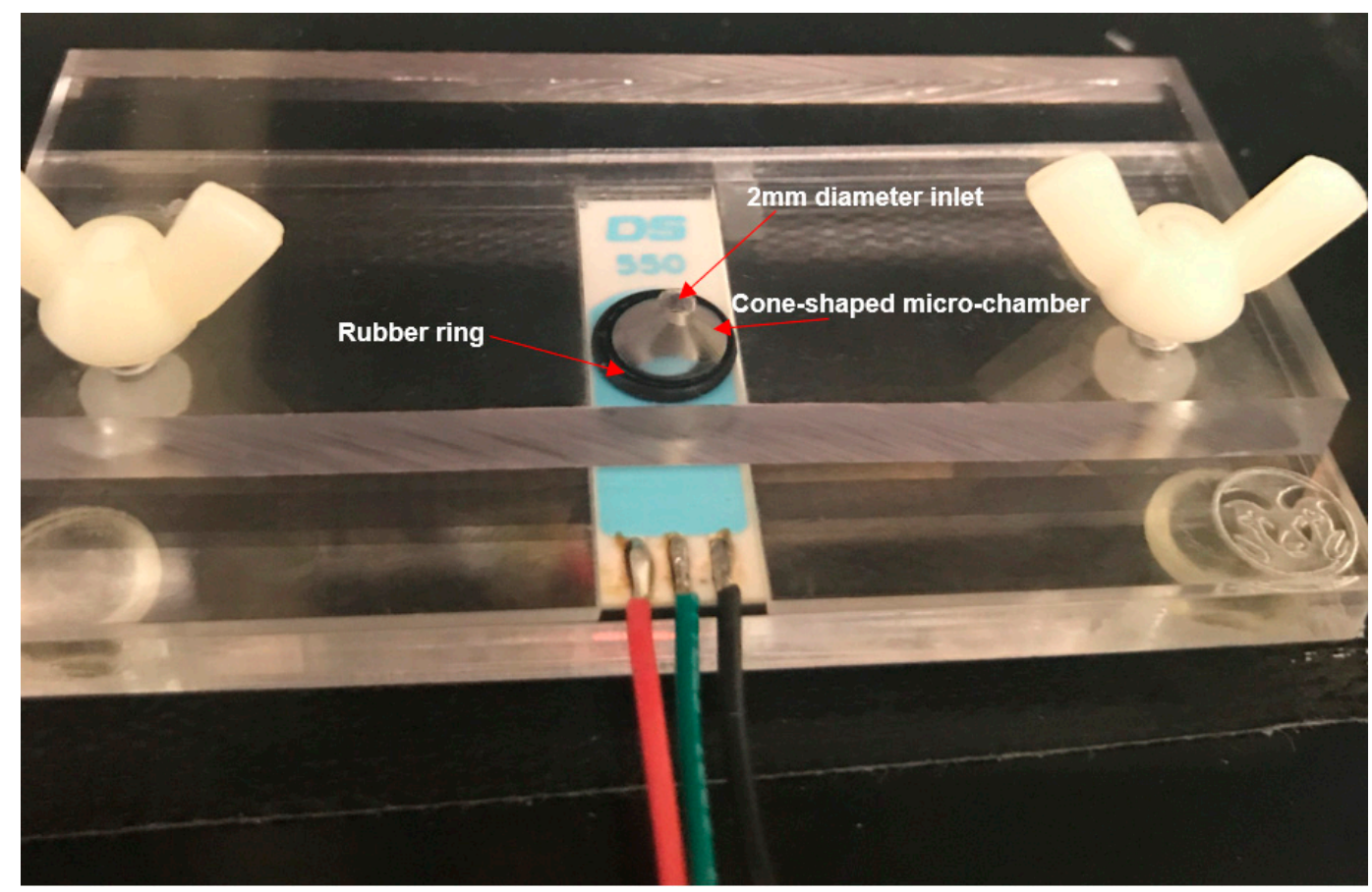

Figure 3. Single channel sample reservoir of $100 \mu \mathrm{L}$ volume max.

\subsection{Electrochemical Instrumentation}

A potentiostat (eDAC, Quadstat EA164H, Colorado Springs, CO) was used to perform all electrochemical measurements. Pulsed cleaning steps were used to clean electrode surface between experiments. The data collected by the potentiostat was analyzed using a set of custom built tools written in Matlab for data calibration and conversion from measured unit in $\mu \mathrm{A} / \mathrm{min}$ to the equivalent respiration rate of $\mathrm{fmol} / \mathrm{s}$.

\subsection{Oxygen Reduction Voltage}

Activation voltages range from $-0.6 \mathrm{~V}$ to $-0.8 \mathrm{~V}$ has been suggested in the literature [36, 37]. However, the actual reduction voltage also depends on electrode size and geometry. To determine the required activation voltage for measuring dissolved oxygen using the sensor in this paper, cyclic voltammetry was performed where the potential was swept from $-1.5 \mathrm{~V}$ to $0.5 \mathrm{~V}$ at $100 \mathrm{mV} / \mathrm{s}$. When deionized (DI) water was tested, a noticeable reduction current was observed in the range $-1 \mathrm{~V}$ to $0.7 \mathrm{~V}$, whereas when sodium sulfite was tested, negligible reduction current was observed. To achieve the least interference from other molecules in the solution, low voltage is preferred; in all experiments, a reduction voltage of $-0.75 \mathrm{~V}$ was used.

\subsection{Negative Control}

In each experiment, negative control was performed by measuring the intrinsic oxygen concentration in the buffer, and the measured current was used as a baseline to determine the amount of oxygen consumption after adding the cells.

\subsection{Calibration}

Sodium sulfite $\left(\mathrm{Na}_{2} \mathrm{SO}_{3}\right)$ was purchased from Eisen-Golden laboratories (Berkeley, California) and was used for measurements as the zero-oxygen concentration. Deionized (DI) water was used for measurements as the saturated dissolved oxygen concentration. To determine the calibration curve and linearity of the sensor, $\mathrm{Na}_{2} \mathrm{SO}_{3}$ was mixed with DI water to produce different oxygen concentrations for generating the calibration data. All $\mathrm{O}_{2}$ concentration measurements by the sensor were validated using calibrated Oakton DO6+ dissolved oxygen meter (Cole-Parmer, Vernon Hills, Illinois). The oxygen sensor responses are plotted against the concentrations (relative percentage of dissolved oxygen) to determine sensor's sensitivity, linearity, and dynamic range. Furthermore, the dissolved oxygen concentration in the MiR05 respiration buffer in $\mu \mathrm{M}$ corresponding to the measured current was calculated at $158 \mu \mathrm{M}$ based on the average barometric pressure of the experiment location (Fort Collins, Colorado $(84.8 \mathrm{kPA})$ ) at the temperature of $38.5{ }^{\circ} \mathrm{C}$, corrected for the slightly lower 
oxygen solubility of the MiR05 respiration buffer (0.92) compared to water, as determined by Rasmussen and Rasmussen [38]. A calibration curve of the measured reduction current versus the relative percentage of dissolved oxygen concentration in DI water and in the MiR05 respiration buffer can be generated.

\subsection{Pulsing Cleaning}

The electrodes were rinsed using DI water and electrochemically cleaned after each test. The pulses used for cleaning were $2 \mathrm{~V}$ peak-to-peak with a sampling rate of $50 \mathrm{mV} / \mathrm{s}$ for one minute. The electrodes were dried using clean wipes after electrochemical cleaning

\subsection{Measurement Setup}

Prior the experiments the WE was covered by a Nafion layer as solid-state electrolyte as well as membrane. The temperature of the medium was maintained at $38.5^{\circ} \mathrm{C}$ by placing the device on the slide warmer during the experiments. The applied potential during all the amperometric experiments was set at $-0.75 \mathrm{~V}$. MiR05 respiration buffer $(90 \mu \mathrm{L})$ was placed in the micro-chamber and overlaid with $90 \mu \mathrm{L}$ Paraffin oil to seal the micro-chamber. The oocyte/embryo was transferred to the microchamber by pipetting through the top oil layer. Oxygen consumption was measured by monitoring the decrease in the oxygen reduction current over time during cell respiration. The change in the measured reduction current over a given period of time was converted to its equivalent oxygen consumption within a defined volume in fmol/s using the calibration curve of the sensor.

Assuming the oxygen concentration gradient within the micro-chamber towards the cell reached its equilibrium state when measurement was taken (i.e. changes in reduction current over time is constant), the defined volume is the volume immediately surrounding the cell inside the micro-chamber. The radius of WE is $2 \mathrm{~mm}$. The cell heights range from 150-200 $\mu \mathrm{m}$ and the cell is placed on WE during the experiments. The defined volume for cell oxygen consumption calculation is the area of the WE time the height of $250 \mu \mathrm{m}$, i.e. $3.14 \mathrm{~mm}^{3}$. Schematic view is shown in Figure 4.

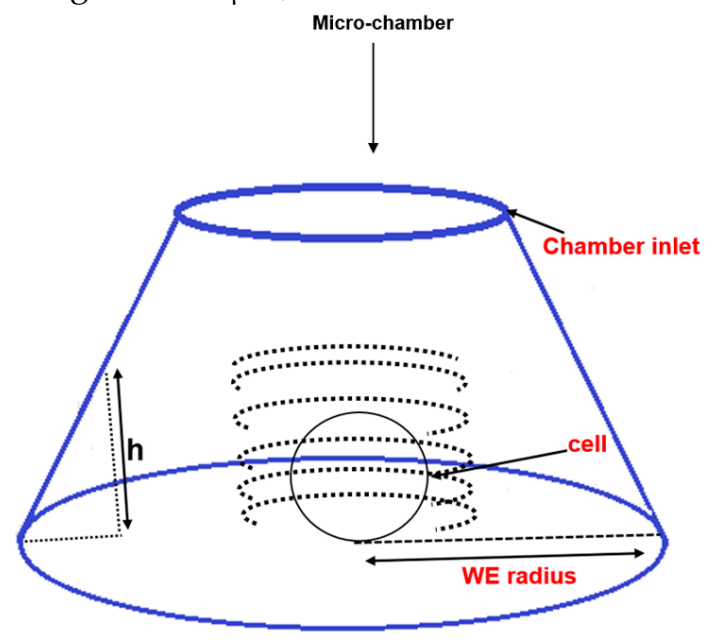

Figure 4. Schematic view of the oxygen consumption measurement for sample located in a cone-shaped microwell. (drawing is not to scale)

Oxygen consumption in $\mathrm{fmol} / \mathrm{s}(\mathrm{OC}(\mathrm{fmol} / \mathrm{s}))$ is defined as:

$$
O C(\mathrm{fmol} / \mathrm{s})=\frac{\Delta C}{\Delta t} \times V
$$

where $\Delta \mathrm{C} / \Delta \mathrm{t}$ is the change in oxygen concentration over a given time period, and $\mathrm{V}$ is the defined volume within the micro-chamber. Equation 4 can be re-written as:

$$
O C(\mathrm{fmol} / \mathrm{s})=\frac{\Delta I / \Delta t}{\Delta I / \Delta C} \times V
$$


where $\Delta \mathrm{I} / \Delta \mathrm{t}$ is the change of reduction current over a given time obtained during experiments, and $\Delta \mathrm{I} / \Delta \mathrm{C}$ is the calibration curve.

\section{Results and Discussion}

\subsection{Sensor Calibration Results}

A calibration curve in Figure 5 was obtained for the sensor when Nafion was used as a solidstate electrolyte as well as membrane. The sensor output current increased with the increase in $\mathrm{O} 2$ concentration in a linear relationship with the correlation coefficient of 0.978 . The error bars represent the standard deviation of ten calibration data points under the same conditions. Variations in pipetting speed and direction and electrochemical crosstalk between electrodes can contribute to variations of the measurement results [26].

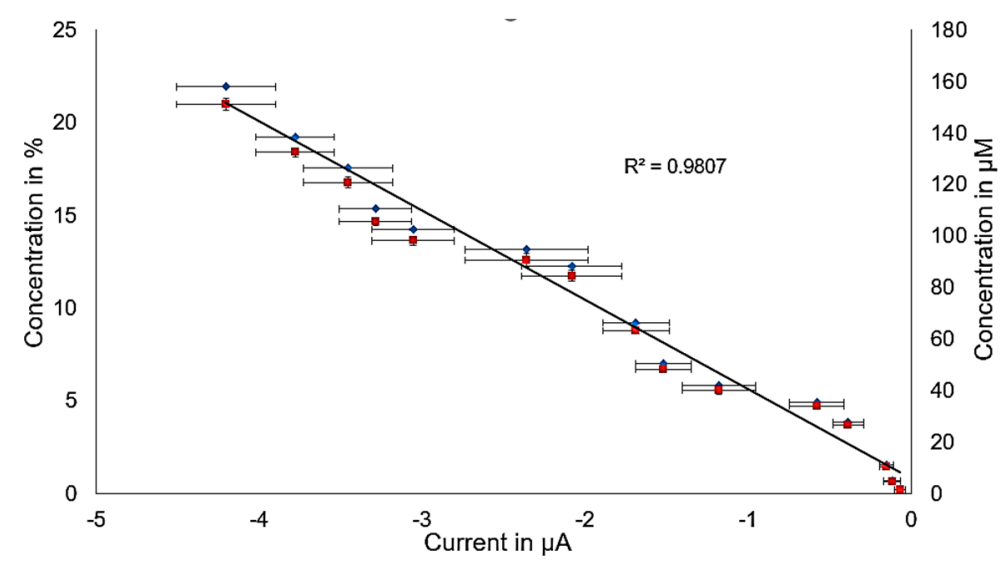

Figure 5. Calibration curve when Nafion was used as solid-state electrolyte.

\subsection{Reproducibility Test}

Deionized (DI) water and $\mathrm{Na}_{2} \mathrm{SO}_{3}$ were added in a sequential cycle to demonstrate the repeatability of the sensor. The sensor showed a good reproducibility with a mean of $3.27 \mu \mathrm{A}$ and a standard deviation of $47 \mathrm{nA}$ in the saturated dissolved oxygen level (21\%) using DI water; and the mean of $27 \mathrm{nA}$ and a standard deviation of $6 \mathrm{nA}$ in the minimum oxygen level (1.7\%) using $\mathrm{Na}_{2} \mathrm{SO}_{3}$. The results also demonstrated a 0\%-to-21\% oxygen response time of 30s as shown in Figure 6.

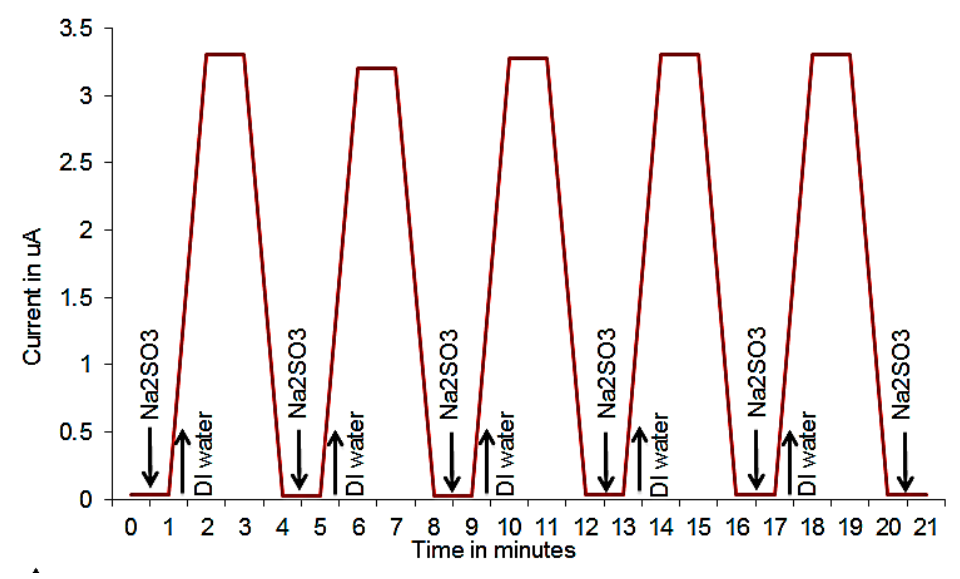

Figure 6. Time response and reproducibility of the Clark sensor 


\subsection{Verification of Airtightness of the Micro-Chamber}

To determine whether the micro-chamber is airtight, $\mathrm{Na}_{2} \mathrm{SO}_{3}$ was loaded into the microchamber, and continuous operations of up to 30 minutes were performed to determine whether oxygen exchange between the micro-chamber and the atmosphere occurred. To test the ability of paraffin oil to seal the micro-chamber, paraffin oil was added to the load cap at the top of the microchamber, and the change in oxygen level inside the micro-chamber was measured and compared with the setup without paraffin oil sealing. The sensor measurement output without the microchamber being sealed shows a change in the measured current across time reflecting a diffusion of atmospheric oxygen into the micro-chamber as shown in Figure 7a. The sensor measurement, when the micro-chamber was sealed by paraffin oil, indicate no oxygen diffusion from the atmosphere as shown in Figure $7 \mathrm{~b}$

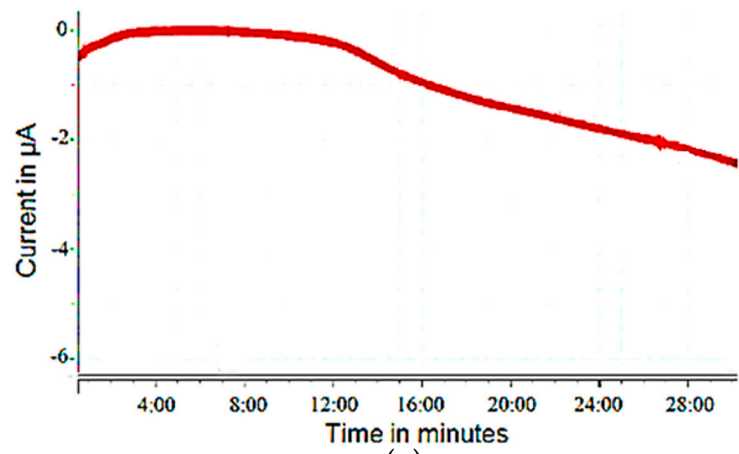

(a)

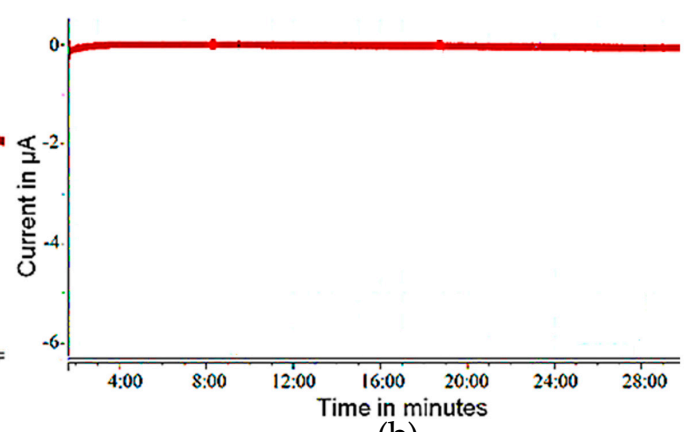

(b)

Figure 7. (a) Time response when $\mathrm{Na}_{2} \mathrm{SO}_{3}$ was tested and no-top sealing, (b) time response when $\mathrm{Na}_{2} \mathrm{SO}_{3}$ was tested with top sealing.

\subsection{Measurement of Basal Mitochondrial Respiration in individual Equine Oocytes}

The vast majority of oxygen consumption by intact living cells is associated with oxidative phosphorylation (OXPHOS) by mitochondria, whereby electrons removed from endogenous metabolic intermediates (oxidation) are transferred through a series of inner membrane protein complexes before reducing oxygen to form water at cytochrome oxidase (respiration). This transfer of electrons is linked to proton translocation across in the inner mitochondrial membrane, which generates a proton gradient that flows through and "powers" the ATP synthase to phosphorylate ADP to ATP. Thus, cellular respiration is coupled to ATP synthesis through generation of an inner membrane proton gradient. The oxygen consumption rate of individual equine oocytes was determined by monitoring the linear decrease in reduction current for 20-30 minutes following stabilization of the electrode signal in $90 \mu \mathrm{L}$ respiration buffer alone as shown in Figure 8a. The measured respiration rate increases accordingly if additional cells are added to the micro-chamber. A nearly-linear increase in basal respiration rate with the increase in the number of cells in the microchamber was achieved as shown in Figure 8b. 

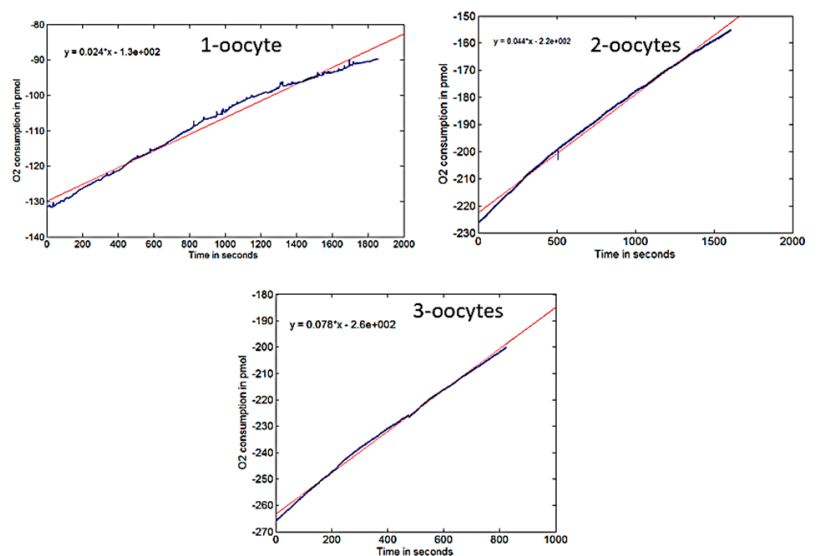

(a)

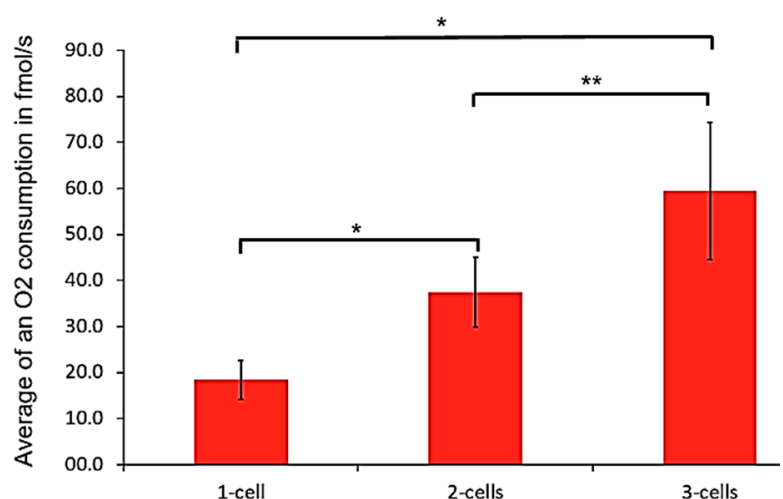

(b)

Figure 8. (a) an example of the difference in the slope of basal respiration of one, two or three bovine oocytes. (b)The average basal respiration of one, two or three bovine oocytes (error bars represent standard deviation (SD) between 7 different experiments). ${ }^{*}$ paired t-test: $\mathrm{p}<0.01$; ${ }^{* *}$ paired $\mathrm{t}$-test: $\mathrm{p}<0.05$.

\subsection{Effect of Oligomycin Addition on Cell Respiration of Equine Oocytes}

Blockade of proton flux through the ATP synthase with oligomycin severely limits the rate of cellular respiration by minimizing dissipation of the inner membrane proton gradient. To confirm that the rate of oxygen consumption detected was associated with oxidative phosphorylation, $1 \mu \mathrm{M}$ oligomycin was added to the microchamber following stabilization of the basal oocyte respiration signal. The results are shown in Figure 9. As expected, the addition of oligomycin reduced respiration to near zero, confirming that essentially all the oxygen consumption being monitored was oocyte mitochondrial respiration. This reflects a high degree of oxidative phosphorylation "coupling" in oocytes, indicated by nearly undetectable levels of "leak" respiration in the absence of ATP synthesis.

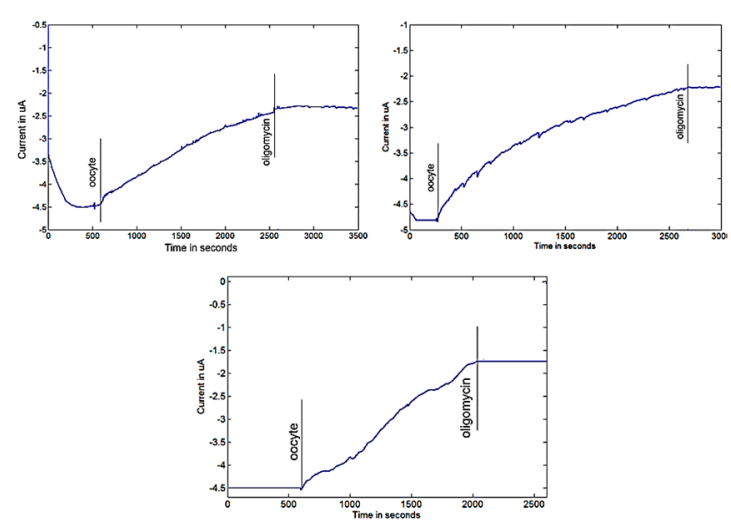

(a)

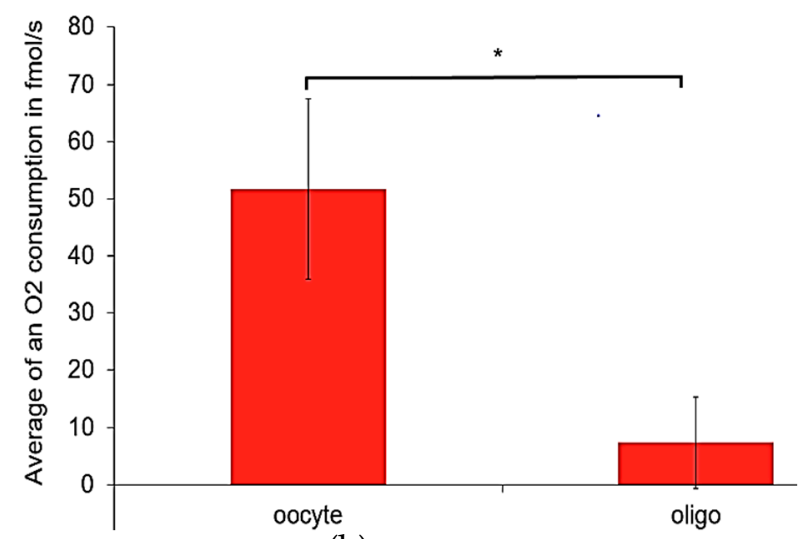

(b)

Figure 9. (a) Amperometry results for three equine oocytes: basal respiration after adding the oocyte followed by the effect of oligomycin. (b) Oxidative phosphorylation activity of equine oocytes: Basal respiration and the amount of respiration after adding oligomycin (error bars are standard deviation (SD) of the measurements of 7 cells). ${ }^{*}$ paired t-test: $\mathrm{p}<0.01$.

\subsection{Effect of FCCP Titration on Cell Respiration of Bovine Oocytes and Embryos}

Importantly, basal oocyte respiration might not represent the cellular mitochondrial respiratory capacity, which can be limited by the basal activity of the ATP synthase (i.e., cellular energy demand). Determining the maximal mitochondrial respiratory capacity is useful for estimating metabolic potential and, when expressed in relation to basal respiration, an index of cellular metabolic activity. Low concentrations of the protonophore "uncoupler" FCCP (1-2 $\mu \mathrm{M})$ are 
routinely used to reveal the maximal respiratory capacity of a cell by removing the limitation of electron flow imposed by mitochondrial membrane potential (proton gradient). However, slightly more FCCP $(3-4 \mu \mathrm{M})$ may inhibit respiratory chain enzymes and decrease respiration in many cell types, resulting in erroneous estimates of cellular respiratory capacity [39]. This effect had not been previously investigated in oocytes, so we performed $1 \mu \mathrm{M}$ titrations of FCCP following stabilization basal oocyte respiration.

The effect of four $1 \mu \mathrm{M}$ titrations of FCCP on bovine oocyte respiration was observed and the result is shown in Figure 10a. FCCP titrations was done after (20-30) min basal respiration and the effect of each titration was measure for $10 \mathrm{~min}$. As expected, the results demonstrate the sensitivity of the electrode to pinpoint the maximal rate of oocyte respiration following $\sim 2 \mu \mathrm{M} \mathrm{FCCP}$, after which an inhibitory effect becomes evident. Figure $10 \mathrm{~b}$ shows that the maximal respiration in bovine embryos is achieved between 1-2 $\mu \mathrm{M}$ FCCP, indicating potential variations in the optimal concentration of FCCP needed to obtain the true maximal respiratory capacity between cell types or developmental stage.

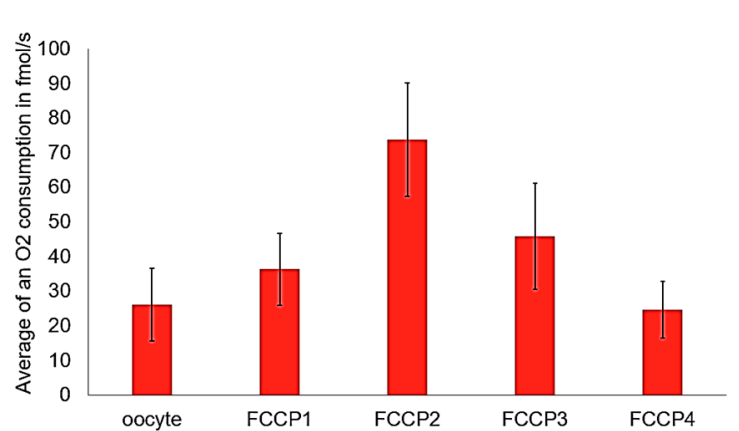

(a)

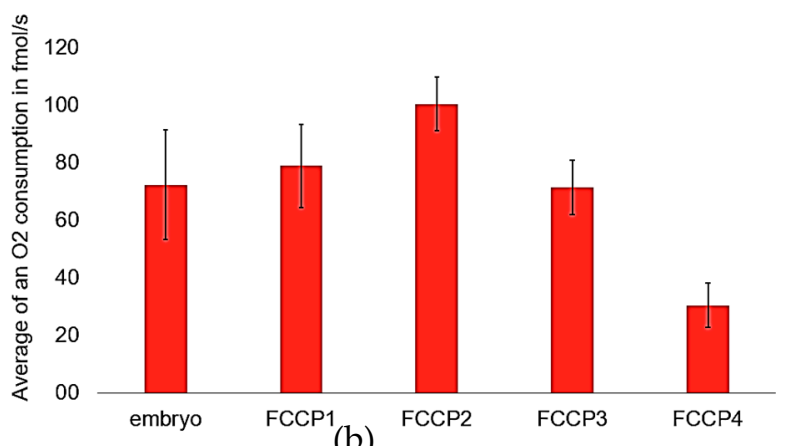

(b)

Figure 10. (a) Oxidative phosphorylation activity of bovine oocytes: Basal respiration and the amount of respiration after four sequential additions of $1 \mu \mathrm{M}$ FCCP at approximately 10 min intervals (error bars are standard deviation(SD) between 10 oocytes) (b) Oxidative phosphorylation activity of bovine embryos: Basal respiration and the amount of respiration after 4 times titration of $1 \mu \mathrm{M} \mathrm{FCCP}$ (error bars are standard deviation(SD) between 6 embryos). paired t-test for all data: $\mathrm{p}<0.05$.

\subsection{Basal Respiration and the Effect of Oligomycin and FCCP Titration on Bovine Oocytes}

Based on the findings in Figure 10, we repeated the experiments to test the effect of oligomycin and FCCP titration on bovine oocytes and embryos. The results of basal respiration and expected inhibitory effects of adding oligomycin $(1 \mu \mathrm{M})$, and recovery of respiration with three $1 \mu \mathrm{M}$ titrations of FCCP on single bovine oocytes and embryos are shown in Figure 11a and Figure 11b, respectively.

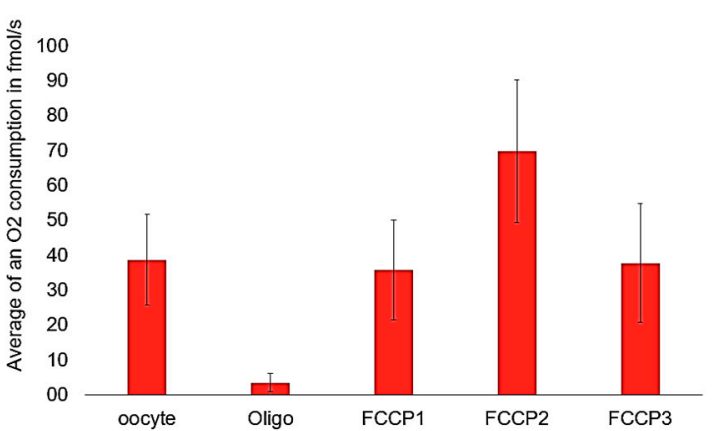

(a)

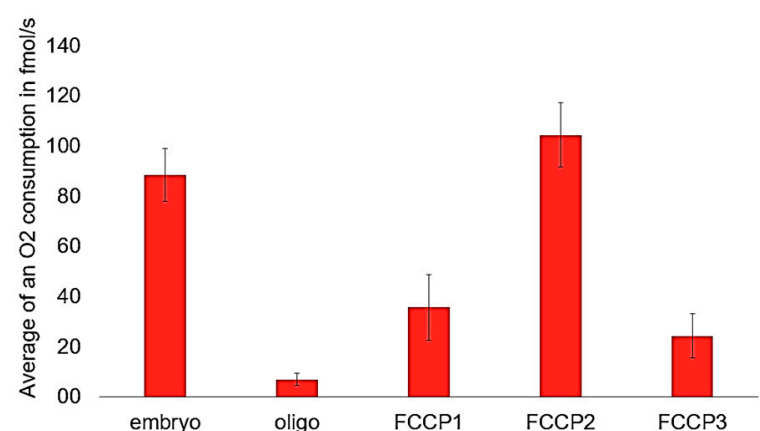

(b)

Figure 11. (a) Oxidative phosphorylation activity of bovine oocytes: Basal respiration and the amount of respiration after adding oligomycin and 3 additive titrations of $1 \mu \mathrm{M}$ FCCP (error bars are standard deviation(SD) between 8 cells). (b) Oxidative phosphorylation activity of embryos: Basal respiration and the 
amount of respiration after adding oligomycin and 3 additive titrations of $1 \mu \mathrm{M} \mathrm{FCCP} \mathrm{(error} \mathrm{bars} \mathrm{are} \mathrm{standard}$ deviation(SD) between 10 embryos). paired t-test for all data pairs: $\mathrm{p}<0.05$

\subsection{Effect of Ethanol on Bovine Oocytes}

Ethanol is a common vehicle for administering oligomycin and FCCP to aqueous solutions for respirometry experiments [39]. To confirm that this vehicle has no effect on oocyte respiration, we evaluated the cumulative effect of multiple $1 \mu \mathrm{L}$ ethanol titrations on bovine oocytes. A negligible effect of three ethanol titrations on oocyte respiration and the expected effects of subsequent FCCP titrations were observed, demonstrating that $\mathrm{EtOH}$ has no toxicity or modulatory effects on respiration of the cells as shown in Figure 12.

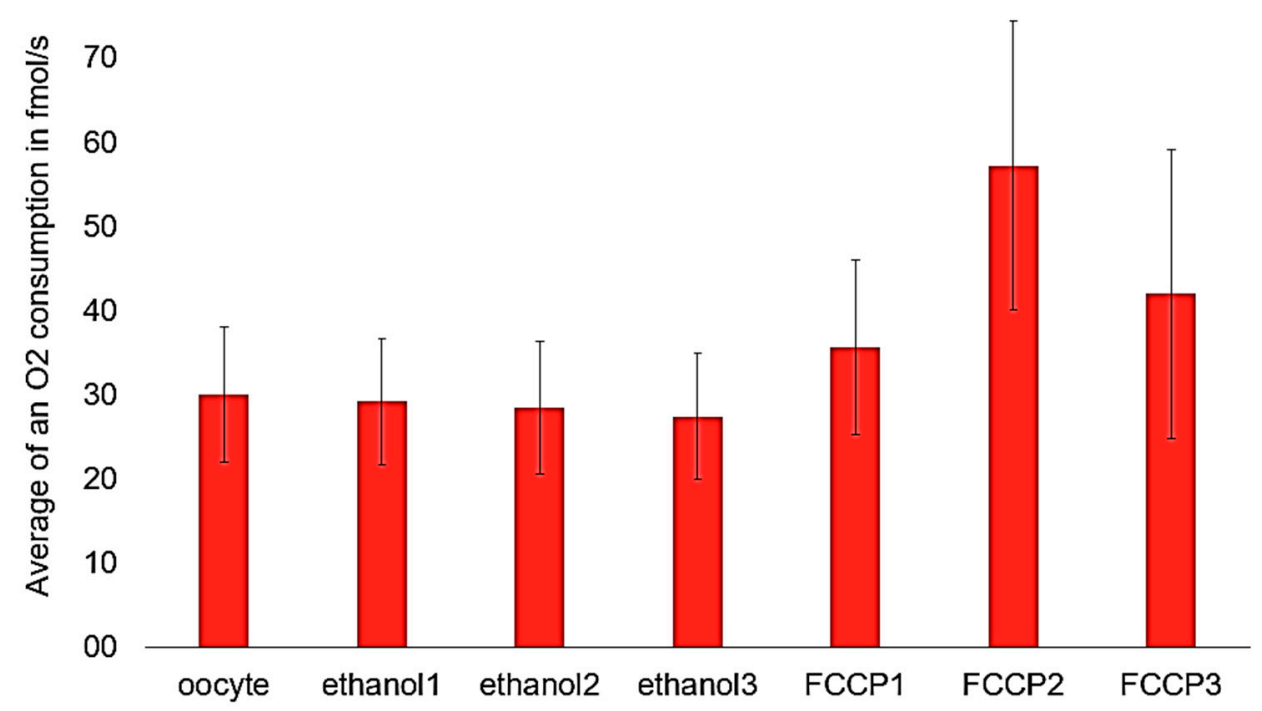

Figure 12. Oxidative phosphorylation activity of bovine oocytes: Basal respiration and the amount of respiration after after 3 times titration of $1 \mu \mathrm{L}$ ethanol followed by 3 times titration of $1 \mu \mathrm{M}$ FCCP (error bars are standard deviation(SD) between 6 cells). paired t-test for all data pairs: $\mathrm{p}<0.05$

\section{Conclusions}

Currently available instruments for measuring respiration are bulky with inconsistent performance and high cost. They are cumbersome and less sensitive than the microchamber system described herein. The commercial devices [18] available for oxygen measurement are expensive and are limited to measuring oxygen up to two samples at a time. Previous studies have demonstrated the value of monitoring oocyte oxygen consumption as a predictors of embryo quality in the context of assisted reproduction technologies [9,10]. Most of these techniques used SECM for the measurement of oxygen concentration where a tip was moved between the cell and bulk solution. These methods assume the cell is in a stable location at the bottom of the chamber during measurement. This assumption is not realistic because the cell can move in the medium during experiments. These techniques also need a precise positioner and motors to achieve an accurate control of the tip's position. In this paper, a three-electrode Clark sensor system was used to investigate oxidative phosphorylation in single bovine oocytes, equine oocytes, and bovine embryos. The sensor was shown to have good linearity, sensitivity, reproducibility, and response time. Unlike the methods using SECM, the method described in this paper measures oxygen consumption right at the immediate vicinity of the cell in the micro-chamber without any moving parts, eliminating the uncertainty of relative positioning in SECM. Validation that respiration measurements were indeed cellular oxidative phosphorylation was demonstrated by expected responses to ATP synthase inhibition and the induction of maximal respiration by titration of the protonophore FCCP. The results in this paper demonstrate that the sensor system can be effectively used to analyze 
mitochondrial function in oocytes to better determine the quality and viability of their development into the blastocyst phase and eventually into embryos.

Moreover, our platform is amenable to the addition of complementary sensors for monitoring of buffer $\mathrm{pH}$ and other metabolites (e.g., glucose, lactate) known to be important during various stages of embryo development [40]. These are currently under development in our laboratory, and may facilitate a multi-sensor metabolic screening approach suitable for clinical and basic scientific applications aimed at understanding and improving cellular metabolism during oocyte maturation and embryo development.

\section{References}

1. Durhuus, J.A., Desler, C., Rasmussen, L.J. Mitochondria in health and disease - 3rd annual conference of society for mitochondrial research and medicine - 19-20 December 2013 - Bengaluru, India. 2015, Mitochondrion 20, 7-12, https://doi.org/10.1016/j.mito.2014.10.004. [PubMed]

2. Park, J., Chang, J.h., Choi, M., Pak, J.J., Lee, D.Y., Pak, Y.K. Microfabricated Clark- type Sensor for Measuring Dissolved Oxygen. IEEE Sensors, Atlanta, GA. 2007, 1412-1415, https://doi.org/10.1109/ICSENS.2007.4388677.

3. Dumollard, R., Duchen, M., Carroll, J. The role of mitochondrial function in the oocyte and embryo. Cur Topics Develop Biol 2007; 77:21-49.https://doi.org/10.1016/S0070-2153(06)77002-8. [PubMed]

4. Pesta, D., Gnaiger, E. High-resolution respirometry: OXPHOS protocols for human cells and permeabilized fibers from small biopsies of human muscle. Methods in Molecular Biology. 2012, 810, 25-58.10.1007/978-161779-382-0_3.

5. Cunningham, C.C., George, D.T. The relationship between the bovine heart mitochondrial adenosine triphosphatase, lipophilic compounds, and oligomycin. J. Biol. Chem. 1975, 250(66), 2036-2044.

6. Chian, R.C., Park, S.E., Park, E.H., Molecular and structural characteristics between immature human oocytes retrieved from stimulated and unstimulated ovaries. In: Gomel V, Leung PCK, eds. In Vitro Fertilization and Assisted Reproduction. 1997, 315-319. Google Scholar

7. Lim, L.H., Chian, R.C. In vitro maturation of human immature oocytes. J Reprod Stem Cell Biotechnol. 2010, 1(2), 169-180.

8. Eichenlaub-Ritter, U., Wieczorek, M., Luke, S., Seidel, T. Age related changes in mitochondrial function and new approaches to study redox regulation in mammalian oocytes in response to age or maturation conditions. Mitochondrian. 2011, 11, 783-796.

9. Tejera, A., Herrero, J., de Los Santos, M.J., Garrido, N., Ramsing, N., Meseguer, M. Oxygen consumption is a quality marker for human oocyte competence conditioned by ovarian stimulation regimens. Fertility and Sterility. 2011, 96(3), 618-623.10.1016/j.fertnstert.2011.06.059.

10. Tejera, A., Castello, D., de Los Santos, M.J., Pellicer, A., Remohi, J., Meseguer, M. Combination of metabolism measurement and a time-lapse system provides an embryo selection method based on oxygen uptake and chronology of cytokinesis timing. Fertility and Sterility. 2016, 106(1), 119126.10.1016/j.fertnstert.2016.03.019

11. Shiku, H., Shiraishi, T., Ohya, H., Matsue, T., Abe, H., Hoshi, H., Kobayashi M. Oxygen consumption of single bovine embryos probed by scanning electrochemical microscopy. Analytical Chemistry. 2001, 73 (15), 3751-3758.10.1021/ac010339j

12. Aizawa, H., Katsumata, T., Komuro, S. Fluorescence oxygen sensor using photo quenching with oxygen molecule. SICE Annual Conference, Kagawa University, Japan. Sept. 17-20, 2007, 1762-1765. 10.1109/SICE.2007.4421269

13. Saharudin, S., Yusof, M., Hamdan, Z., Zolkipli, M., Abdullah, W., Herman, S. Performance improvement of optical fiber oxygen sensor detection scheme incorporating narrow bandpass emission optical filter. IEEE.2015, 10.1109/ICSENS.2015.7370184 
14. Maerz, H., Buchholz, R., Emmrich, F., Fink, F., Geddes, C., Pfeifer, L., Raabe, F., Scheper, T.H., Ulrich, E., Marx, U. Applying fiber optical methods for toxicological testing in vitro. Proceeding of SPIE-The International Society for optical engineering. 1999, 3603, 228-236.

15. Edwards, M.A., Martin, S., Whitworth, A.L., Macpherson, J.V., Unwin, P.R. Scanning electrochemical microscopy: principles and applications to biophysical systems. Physiol Meas. 2006, 27, R63-R108.

16. Sugimura, S., Matoba, S., Hashiyada, Y., Aikawa, Y., Ohtake, M., Matsuda, H., Kobayashi, S., Konishi, K., Imai, K. Oxidative phosphorylation-linked respiration in individual bovine oocytes. J Reprod Develop. 2012, 58(6), 636-641.

17. Cussler, L.E. Diffusion: Mass Transfer in Fluid Systems, $3^{\text {rd }}$ ed. Cambridge University Press, New York. 1984.

18. Bodri, D., Kato, R., Kondo, M., Hosomi, N., Katsumata, Y., Kawachiya, S., Matsumoto, T. Time-lapse monitoring of zona pellucida-free embryos obtained through in vitro fertilization: a retrospective case series. Fertil Steril. 2015 May, 103(5), e35. 10.1016/j.fertnstert.2015.02.016

19. Macpherson, J.V., Unwin, P.R. Scanning electrochemical microscope induced dissolution: theory and experiment for silver chloride dissolution in aqueous solution without supporting electrolyte. J. Phys. Chem.1996, 100 (50), 19475-19483. 10.1021/jp9614862

20. Liu, H. Y., Fan, F.R.F, Lin, C. W., Bard, A. J. Scanning electrochemical and tunneling ultramicroelectrode microscope for high-resolution examination of electrode surfaces in solution. J. Am. Chem. Soc. 1986, 108 (13), 3838-3839. 10.1021/ja00273a054

21. Kranz, C., Ludwig, M., Gaub, H. E., Schuhmann, W. Lateral deposition of polypyrrole lines by the scanning electrochemical microscope. Adv. Mater.1995. 7, 38-40.10.1002/adma.19950070106

22. Clark, L. C., Jr. Monitor and Control of Blood and Tissue Oxygen Tensions. Trans. Am. Soc. Art. Int. Organs. 1956, 2, 41-48.

23. Compton, G., Laborda, E., Wand, K. Understanding Voltammetry. 2nd Edition. Imperial College Press. 2011.

24. Ramamoorthy, R., Dutta, P.K., Akbar, S.A. Oxygen sensors: Materials, methods, designs and applications. Journal of Materials Science. 2003, 38, 4271-4282. 10.1023/A:1026370729205

25. Jobst, G., Urban, G., Jachimowicz, A., Kohl, F., Tilado, O., Lettenbichler, I., Nauer, G. Thin-film Clark-type oxygen sensor based on novel polymer membrane systems for in vivo and biosensor applications. Biosensors and Bioelectronics. 1993, 8, 123-128.

26. Wu, C., Yasukawa, T., Shiku, H., Matsue,T. 2005 Fabrication of miniature Clark oxygen sensor integrated with microstructure. . Sensors and Actuators B. 2005, 110, 342-349.

27. Yang, Z., Sasaki, S., Karube, I., Suzuki, H. Fabrication of oxygen electrode arrays and their incorporation into electrodes for measuring biochemical oxygen demand. Anal. Chim. Acta. 1997, 357, 41-49.

28. Jenkins, J., Dmitriev, R., Morten, K., McDermott, K., Papkovsky, D. Oxygen-sensing scaffolds for 3dimensional cell and tissue culture. Acta Materialia Inc. Published by Elsevier Ltd. 2015, 16, 126-35.

29. Miyahara, Y., Tsukada, K., Watanabe, Y., Shibata, Y. Long-life planar oxygen sensor. Sensors and Actuators: B. Chemical. 1994, 20(2-3), 89-94. http://dx.doi.org/10.1016/0925-4005(93)01188-A

30. Suzuki, H., Sugama, A., Kojima, N., Micromachined Clark oxygen electrode. Sens. Actuators B. 1993, 10, 91-98.

31. McLaughlin, G.W., Braden, K., Franc, B., T.A. Kovacs, G. Microfabricated solid-state dissolved oxygen sensor. Sensors and Actuators B: Chemical. 2002, 138-148.

32. Obeidat, Y., Chen, T., Characterization of an O2 Sensor Using Microelectrodes. IEEE sensors. Orlando, FL, Oct. 30 - Nov. 2, 2016. https://doi.org/10.1109/ICSENS.2016.7808460

33. Niazi, A., Anthony, C. Development of Oxygen Sensor by Integrating the Low Cost Printed Circuit Board Technology and Solid Electrolyte Membrane. Proceedings of the International Conference on Biomedical Engineering and Systems Prague, Czech Republic. August 14-15, 2014, paper No. 137.

34. France, B., Peixoto, N., Giovangrandi, L.,McLaughlin, G., T.A.Kovacs, G. Measurerment of Changes in tissue metabolism using a Clark-type oxygen sensor. Solid-State Sensor, Actuator and Microsystems Workshop. 2002.

35. De La Torre-Sanchez JF, Preis K, Seidel GE Jr. Metabolic regulation of in-vitro-produced bovine embryos. I. Effects of metabolic regulators at different glucose concentrations with embryos produced by semen from different bulls. Reprod Fertil Dev. 2006, 18(5), 585-96. [ㅁubMed] 
36. Maclay, G.J., Buttner, W.J, Stetter, J.R. Microfabricated Amperometric Gas Sensors. IEEE Transactions on Electron Devices. June 1988, 35, 793-799. https://doi.org/10.1109/16.2533

37. Suzuki, H., Sugama, A., Kojima,N. Miniature Clark-type oxygen electrode with a three-electrode configuration. Sens. Actuators B. 1990, 2, 297-303.

38. Rasmussen, H.N., Rasmussen, U.F. Oxygen solubilities of media used in electrochemical respiration measurements. Analyt Biochem. 2003, 319, 105-113.

39. Steinlechner-Maran R, Eberl T, Kunc M, Margreiter R, Gnaiger E. Oxygen dependence of respiration in coupled and uncoupled endothelial cells. Am J Physiol. 1996, 271, C2053-C2061. [PubMed]

40. Thompson, J.G., Partridge, R.J., Houghton, F.D., Cox, C.I., Leese, H.J. Oxygen uptake and carbohydrate metabolism by in vitro derived bovine embryos. Journal of Reproduction and Fertility. 1996, 106(2), 299 306. [PubMed] 